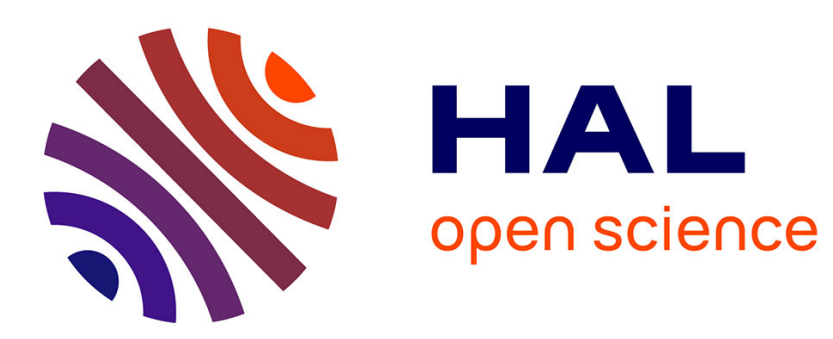

\title{
Uncertainties assessment in global sensitivity indices estimation from metamodels
}

\author{
Alexandre Janon, Maëlle Nodet, Clémentine Prieur
}

\section{To cite this version:}

Alexandre Janon, Maëlle Nodet, Clémentine Prieur. Uncertainties assessment in global sensitivity indices estimation from metamodels. 2011. inria-00567977v2

\section{HAL Id: inria-00567977 https://hal.inria.fr/inria-00567977v2}

Preprint submitted on 15 Nov 2011 (v2), last revised 15 Jun 2012 (v3)

HAL is a multi-disciplinary open access archive for the deposit and dissemination of scientific research documents, whether they are published or not. The documents may come from teaching and research institutions in France or abroad, or from public or private research centers.
L'archive ouverte pluridisciplinaire HAL, est destinée au dépôt et à la diffusion de documents scientifiques de niveau recherche, publiés ou non, émanant des établissements d'enseignement et de recherche français ou étrangers, des laboratoires publics ou privés. 


\title{
Uncertainties assessment in global sensitivity indices estimation from metamodels
}

\author{
Alexandre Janon, Maëlle Nodet, Clémentine Prieur \\ Joseph Fourier University, Laboratoire Jean Kuntzmann, MOISE team, \\ BP 53, 38041 Grenoble Cedex, France
}

November 14, 2011

\begin{abstract}
Global sensitivity analysis is often impracticable for complex and resource intensive numerical models, as it requires a large number of runs. The metamodel approach replaces the original model by an approximated code that is much faster to run. This paper deals with the information loss in the estimation of sensitivity indices due to the metamodel approximation. A method for providing a robust error assessment is presented, hence enabling significant time savings without sacrificing on precision and rigor. The methodology is illustrated on two different types of metamodels: one based on reduced basis, the other one on RKHS interpolation.
\end{abstract}

Keywords: sensitivity analysis Sobol indices bootstrap method Monte Carlo method metamodels.

\section{Contents}

1 Review of sensitivity indices $\quad 4$

1.1 Definition .................... 4

1.2 Monte-Carlo estimator . . . . . . . . . . . . . 5

2 Quantification of the two types of error in index estimation 6

2.1 Sampling error : bootstrap confidence intervals . . . . . . 6

2.2 Metamodel error in index estimation . . . . . . . . . . 7

2.3 Metamodel error in index estimation: a smoothed alternative 10

2.4 Combined confidence intervals . . . . . . . . . . . . . . 11

3 Applications $\quad 12$

3.1 Application to a reduced basis metamodel . . . . . . . . . 12

3.1.1 Model set-up . . . . . . . . . . . . . . . . 12

3.1.2 Empirical coverage of combined confidence intervals . 14

3.1.3 Convergence benchmark . . . . . . . . . . . . . 14

3.1.4 Choice of $n$ and $N \ldots \ldots \ldots \ldots$ 
3.1.5 Full-scale example . . . . . . . . . . . . 16

3.2 Application to a RKHS interpolation metamodel . . . . . . 17

3.2.1 RKHS interpolation and error bound . . . . . . . 17

3.2 .2 Application to Ishigami function . . . . . . . . . . 18

\section{Introduction}

Many mathematical models use a large number of poorly-known parameters as inputs. The impact of parameter uncertainty on the model output is important for the user of these models. This problem can be tackled by considering the uncertain input parameters as random variables, whose probability distribution reflects the practitioner's belief in the precision of the parameter values. Model output, as function of the model inputs, is then a random variable. Its probability distribution, uniquely determined by the model and the distribution of the inputs, can give detailed and valuable information about the behavior of the output when input parameters vary: range of attained values, mean value and dispersion about the mean, most probable values (modes), etc.

Sensitivity analysis aims to identify the sensitive parameters, that is, parameters for which a small variation implies a large variation of the model output. In global sensitivity analysis, one makes use of the probability distribution of the outputs to define (amongst other sensitivity measures) sensitivity indices (also known as Sobol indices). The sensitivity index of an output with respect to an input variable is the fraction of output variance which can be "explained" by the variation of the input variable, either alone (one then speaks about main effect), or in conjunction with other input variables (total effect). This way, input variables can be sorted by the order of importance they have on the output. One can also consider the proportion of variance due to the variation of groups of two or more inputs, although main effects and total effects are generally sufficient to produce a satisfying sensitivity analysis; see, e.g., $[11,18]$ for more information about uncertainty and sensitivity analysis.

Once these indices have been defined, the question of their effective calculation remains open. For most models, an exact, analytic computation is not attainable (even expressing an output as an analytic function of the inputs is infeasible) and one has to use numerical approximations.

A robust, popular way to obtain such approximations is Monte Carlo estimation. This method simulates randomness in inputs by sampling a large number of parameter values (from the selected input distribution). The model output is then computed for each sampled value of the parameters. This way, one obtains a sample of outputs, under the conjugate action of the model and the input distribution. A suitable statistical estimator can then be applied to form a numerical estimate of the sensitivity index based on the 
sample of outputs. The Monte Carlo approach to Sobol indices computation is described in [22], together with improvements in [12, 17].

A major drawback of the Monte Carlo estimation is that a large number of model outputs have to be evaluated for the resulting approximation of the sensitivity index to be accurate enough to be useful. In complex models, in which a simulation for one single value of the parameters may take several minutes, using these methods "as-is" is impracticable. In those cases, one generally makes use of a surrogate model (also known as reduced model, emulator, metamodel or response surface). The surrogate model has to approximate the original model (called the full model) well, while being many times faster to evaluate. The sensitivity index is then calculated via a sample of outputs, each generated by a call to the surrogate model, thus accelerating the overall computation. The aim of this paper is to quantify accuracy loss due to the use of a metamodel combined to a Monte-Carlo method to compute sensitivity indices.

The sensitivity index produced by Monte Carlo estimation with a surrogate model is tainted by a twofold error. Firstly, our Monte-Carlo sampling procedure assimilates the whole (generally infinite) population of possible inputs with the finite, randomly chosen, sample; this produces sampling, or Monte-Carlo error. Secondly, using a surrogate model biases the estimation of the Sobol index, as what is actually estimated is sensitivity of surrogate output, and not the full one; we call this bias the metamodel error.

In order to make a rigorous sensitivity analysis, it is important to assess the magnitude of these two combined errors on the estimated sensitivity indices. Such assessment can also be used to help with the choice of correct approximation parameters (Monte-Carlo sample size and metamodel fidelity) to achieve a desired precision in estimated indices.

Sampling error can be classically estimated for a moderate cost by using bootstrap resampling $([8,1])$. Based on statistical estimation theory, the bootstrap technique involves the generation of a sample of sensitivity index estimator replications, whose empirical distribution serves as approximation of the true (unknown) estimator distribution, in order to produce asymptotic confidence intervals that give good results in many practical cases.

Some surrogate models come with error bounds, that is, computable (or at least estimable) upper bounds on the error between the original and the surrogate outputs. The reduced basis (RB) method $([15,10,24,9])$ is a method leading to such rigorously certified metamodels; it is applicable when the original model is a discretization of a partial differential equation (PDE) depending on the input parameters. Kriging ([19]) - also known as Gaussian process metamodelling, which is equivalent to RKHS (reproducing kernel Hilbert space) interpolation ([20]) - also provides error indicators. In contrast to the RB method, Kriging/RKHS interpolation only requires "blackbox" training data, i.e. a (finite) set of input-output pairs from the original model. This makes it more versatile and easier to use, at the expense 
of rigor in the error bounds and quality in the approximation.

A variation on the bootstrap, which addresses sampling error as well as metamodel error, has been proposed in [23]; [14] has developed a methodology in Kriging metamodels, and the paper by [2] makes use of the reducedbasis output error bound to certify computation of the expectation and the variance of a model output with neglected sampling error.

The advantages of our approach are: its rigorousness (the impact of the use of a surrogate model is provably bounded); its efficiency (our bounds are rather sharp, and go to zero when metamodel errors decrease); its clear separation between estimation (sampling) and metamodel error; its moderate computational requirements (time is better spent on making a precise computation than at measuring precision) and its versatility with respect to metamodel choice (the user can choose any metamodel that comes with computable error bound or error indicator). In other words, our method allows us to estimate sensitivity indices by using a metamodel which greatly speeds up computation times, while rigorously keeping track of the precision of the estimation.

This paper is organized as follows: in the first part, we describe the prerequisites for our approach: we give the definition of the standard Monte Carlo estimator of the sensitivity indices of interest; in the second part, we present our confidence interval estimation technique for the sensitivity index, which accounts for the two sources of error described earlier (sampling and metamodel). In the third part, we present applications of our method for reduced-basis and RKHS/Kriging interpolation metamodels.

\section{Review of sensitivity indices}

\subsection{Definition}

In order to define sensitivity indices, we choose a probability distribution for the input variables, considering each input variable $X_{i}(i=1, \ldots, p)$ as a random variable with known distribution; the model output $Y=f\left(X_{1}, \ldots, X_{p}\right)$ (assumed to be square-integrable, non a.s. constant and scalar: multiple outputs can be treated separately) is thus for $\mathbf{X}=\left(X_{1}, \ldots, X_{p}\right)$ a $\sigma(\mathbf{X})$ measurable random variable (assuming that $f$ is a $\sigma(\mathbf{X})$-measurable function). We further assume that the $X_{i}$ 's are mutually independent. We also fix throughout all this paper an input variable of interest $1 \leq i \leq p$. We define the first-order main effect of $X_{i}$ on $Y$ by:

$$
S_{i}=\frac{\operatorname{Var} \mathbf{E}\left(Y \mid X_{i}\right)}{\operatorname{Var} Y}
$$

$S_{i}$ is the sensitivity index in which we are interested in this paper but other indices (total effect, high-order effects) exist and our methodology can readily be extended to these indices. 


\subsection{Monte-Carlo estimator}

We are interested in the following Monte-Carlo estimator for $S_{i}([12,17])$ : a sample size $N \in \mathbf{N}$ being given, let $\left\{\mathbf{X}^{k}\right\}_{k=1, \ldots, N}$ and $\left\{\mathbf{X}^{\prime k}\right\}_{k=1, \ldots, N}$ be two random i.i.d. samples of size $N$ each, drawn from the distribution of the input vector $\mathbf{X}$.

For $k=1, \ldots, N$, we note:

$$
y_{k}=f\left(\mathbf{X}^{k}\right)
$$

and:

$$
y_{k}^{\prime}=f\left(X_{1}^{\prime k}, \ldots, X_{i-1}^{\prime k}, X_{i}^{k}, X_{i+1}^{\prime k}, \ldots, X_{p}^{\prime k}\right)
$$

The Monte-Carlo estimator of $S_{i}$ is then given by:

$$
\widehat{S_{i}}=\frac{\overline{y y^{\prime}}-\bar{y} \times \overline{y^{\prime}}}{\overline{y^{2}}-\bar{y}^{2}}
$$

where, for any vector $v=\left(v_{1}, \ldots, v_{N}\right)$,

$$
\bar{v}=\frac{1}{N} \sum_{k=1}^{N} v_{i}
$$

It can be shown that $\widehat{S_{i}}$ is a strongly consistent estimator of $S_{i}$.

Remark: our methodology can be extended to higher order Sobol indices by choosing primed output sample $\left\{y_{k}^{\prime}\right\}$ appropriately. More specifically, for a subset of variables $I \subset\{1, \ldots, p\}$, the closed index with respect to $I$, defined by:

$$
S_{I}=\frac{\operatorname{Var} \mathbf{E}\left(Y \mid\left(X_{i}\right)_{i \in I}\right)}{\operatorname{Var} Y}
$$

is estimated by using:

$$
y_{k}^{\prime}=f\left(\mathbf{X}_{I}^{\prime k}\right), \text { where } X_{I, i}^{\prime k}=\left\{\begin{array}{l}
X_{i}^{k} \text { if } i \in I \\
X_{i}^{\prime k} \text { else }
\end{array} .\right.
$$

and standard high-order indices can be treated by subtracting the effects of the proper subsets of $I$.

\section{Quantification of the two types of error in index estimation}

We now present our method for estimating the two types of error that occur in Monte-Carlo sensitivity index estimation on a reduced-basis metamodel. In Section 2.1, we review the bootstrap, which we will use for the treatment of sampling error. In Sections 2.2 and 2.3, we show two approaches for taking metamodel error in account. 


\subsection{Sampling error : bootstrap confidence intervals}

Sampling error, due to the Monte-Carlo evaluation of the variances in (1), can be quantified through an approximate confidence interval calculated using bootstrap ([1]).

We use the bias-corrected $(\mathrm{BC})$ percentile method presented in $[6,7]$. The principle of this method can be summed up the following way: let $\hat{\theta}\left(X_{1}, \ldots, X_{n}\right)$ be an estimator for an unknown parameter $\theta$ in a reference population $\mathcal{P}$. We generate a random i.i.d. $n$-sample $\left\{x_{1}, \ldots, x_{n}\right\}$ from $\mathcal{P}$, then we repeatedly, for $b=1, \ldots, B$, randomly draw $\left\{x_{1}[b], \ldots, x_{n}[b]\right\}$ with replacement from this sample and get a replication of $\widehat{\theta}$ by computing $\widehat{\theta}[b]=\widehat{\theta}\left(x_{1}[b], \ldots, x_{n}[b]\right)$. This way we obtain a set $\mathcal{R}=\{\widehat{\theta}[1], \ldots, \widehat{\theta}[B]\}$ of replications of $\widehat{\theta}$.

We now show how this sample can be used to estimate a confidence interval for $\theta$. We denote by $\Phi$ the standard normal cdf:

$$
\Phi(z)=\frac{1}{\sqrt{2 \pi}} \int_{-\infty}^{z} \exp \left(-\frac{t^{2}}{2}\right) \mathrm{d} t
$$

and by $\Phi^{-1}$ its inverse.

Using $\mathcal{R}$ and the point estimate $\widehat{\theta}=\widehat{\theta}\left(x_{1}, \ldots, x_{n}\right)$, a "bias correction constant" $z_{0}$ can be estimated:

$$
\widehat{z_{0}}=\Phi^{-1}\left(\frac{\#\{\widehat{\theta}[b] \in \mathcal{R} \text { s.t. } \widehat{\theta}[b] \leq \widehat{\theta}\}}{B}\right)
$$

where $\# A$ denotes the number of elements in the set $A$.

Then, for $\beta \in] 0 ; 1[$, we define the "corrected quantile estimate" $\widehat{q}(\beta)$ :

$$
\widehat{q}(\beta)=\Phi\left(2 \widehat{z_{0}}+z_{\beta}\right)
$$

where $z_{\beta}$ satisfies $\Phi\left(z_{\beta}\right)=\beta$.

The central BC bootstrap confidence interval of level $1-\alpha$ is then estimated by the interval whose endpoints are the $\widehat{q}(\alpha / 2)$ and $\widehat{q}(1-\alpha / 2)$ quantiles of $\mathcal{R}$.

The BC bootstrap procedure is known to be robust to non-normality of $\widehat{\theta}$. The key advantage of bootstrapping our sensitivity estimators is that we do not require supplementary model evaluations to estimate a confidence interval; hence the computational overhead for getting a confidence interval (versus pointwise estimation only) remains quite modest.

\subsection{Metamodel error in index estimation}

We now denote by $\tilde{f}: \mathcal{P} \subseteq \mathbf{R}^{p} \rightarrow \mathbf{R}$ the metamodel output approximating $f: \mathcal{P} \rightarrow \mathbf{R}$, and by $\varepsilon$ the pointwise error bound that certifies the metamodel approximation, i.e. we have:

$$
|f(\mathbf{x})-\tilde{f}(\mathbf{x})| \leq \varepsilon(\mathbf{x}) \quad \forall \mathbf{x} \in \mathcal{P}
$$


For a pair of samples $\left(\left\{\mathbf{X}^{k}\right\}_{k=1, \ldots, N},\left\{\mathbf{X}^{\prime k}\right\}_{k=1, \ldots, N}\right)$ of inputs, we can use our metamodel output $\widetilde{f}$ and our metamodel error bound $\varepsilon$ to compute, for $k=1, \ldots, N$ :

$$
\widetilde{y}_{k}=\widetilde{f}\left(\mathbf{X}^{k}\right), \quad \widetilde{y}_{k}^{\prime}=\widetilde{f}\left(X_{1}^{\prime k}, \ldots, X_{i-1}^{\prime k}, X_{i}^{k}, X_{i+1}^{\prime k}, \ldots, X_{p}^{\prime k}\right)
$$

and:

$$
\varepsilon_{k}=\varepsilon\left(\mathbf{X}^{k}\right), \quad \varepsilon_{k}^{\prime}=\varepsilon\left(X_{1}^{\prime k}, \ldots, X_{i-1}^{\prime k}, X_{i}^{k}, X_{i+1}^{\prime k}, \ldots, X_{p}^{\prime k}\right)
$$

In this section, we find accurate, explicitly and efficiently computable bounds $\widehat{S}_{i}^{m}$ and $\widehat{S}_{i}^{M}$, depending only on $\widetilde{y}_{k}, \widetilde{y}_{k}^{\prime}, \varepsilon_{k}$ and $\varepsilon_{k}^{\prime}$ so that:

$$
\widehat{S}_{i}^{m} \leq \widehat{S}_{i} \leq \widehat{S}_{i}^{M}
$$

In other words, we want lower and upper bounds on the full model based sensitivity index estimator $\widehat{S}_{i}$ computable from surrogate model calls.

Let:

$$
R\left(a ; \mathbf{y}, \mu, \mu^{\prime}\right)=\sum_{k=1}^{N}\left(y_{k}^{\prime}-\left(a\left(y_{k}-\mu\right)+\mu^{\prime}\right)\right)^{2}
$$

where $\mathbf{y}=\left(y_{1}, \ldots, y_{N}, y_{1}^{\prime}, \ldots, y_{N}^{\prime}\right)$ and $\mu, \mu^{\prime} \in \mathbf{R}$.

By setting first derivative of $R$ with respect to $a$ to zero, making use of the convexity of $R\left(\cdot ; \mathbf{y}, \bar{y}, \bar{y}^{\prime}\right)$ and using the definition (4) of $\widehat{S_{i}}$, one easily shows that:

$$
\widehat{S}_{i}=\underset{a \in \mathbf{R}}{\operatorname{argmin}} R\left(a ; \mathbf{y}, \bar{y}, \bar{y}^{\prime}\right) .
$$

In other words, $\widehat{S}_{i}$ is the slope of the linear least squares regression of the $\left\{y_{k}^{\prime}\right\}_{k}$ on the $\left\{y_{k}\right\}_{k}$.

Define:

$R_{\text {inf }}\left(a ; \widetilde{\mathbf{y}}, \varepsilon, \mu, \mu^{\prime}\right)=\sum_{k=1}^{N}\left\{\inf _{z \in\left[\widetilde{y}_{k}-\varepsilon_{k} ; \widetilde{y}_{k}+\varepsilon_{k}\right], z^{\prime} \in\left[\widetilde{y}_{k}^{\prime}-\varepsilon_{k}^{\prime}, \widetilde{y}_{k}^{\prime}+\varepsilon_{k}^{\prime}\right]}\left(z^{\prime}-\left(a(z-\mu)+\mu^{\prime}\right)\right)^{2}\right\}$

and:

$R_{\text {sup }}\left(a ; \widetilde{\mathbf{y}}, \boldsymbol{\varepsilon}, \mu, \mu^{\prime}\right)=\sum_{k=1}^{N}\left\{\sup _{z \in\left[\widetilde{y}_{k}-\varepsilon_{k} ; \widetilde{y}_{k}+\varepsilon_{k}\right], z^{\prime} \in\left[\widetilde{y}_{k}^{\prime}-\varepsilon_{k}^{\prime} ; \widetilde{y}_{k}^{\prime}+\varepsilon_{k}^{\prime}\right]}\left(z^{\prime}-\left(a(z-\mu)+\mu^{\prime}\right)\right)^{2}\right\}$

where $\widetilde{\mathbf{y}}=\left(\widetilde{y}_{1}, \ldots, \widetilde{y}_{N}, \widetilde{y}_{1}^{\prime}, \ldots, \widetilde{y}_{N}^{\prime}\right), \boldsymbol{\varepsilon}=\left(\varepsilon_{1}, \ldots, \varepsilon_{N}, \varepsilon_{1}^{\prime}, \ldots, \varepsilon_{N}^{\prime}\right)$.

It is clear that:

$$
R_{\text {inf }}\left(a ; \widetilde{\mathbf{y}}, \varepsilon, \mu, \mu^{\prime}\right) \leq R\left(a ; \mathbf{y}, \mu, \mu^{\prime}\right) \leq R_{\text {sup }}\left(a ; \widetilde{\mathbf{y}}, \varepsilon, \mu, \mu^{\prime}\right) \quad \forall a, \mu, \mu^{\prime} \in \mathbf{R}
$$

Note that $R, R_{\text {inf }}$ and $R_{\text {sup }}$ are quadratic polynomials in $a$. We name $\alpha, \beta, \gamma, \alpha_{\text {inf }}, \beta_{\text {inf }}, \gamma_{\text {inf }}, \alpha_{\text {sup }}, \beta_{\text {sup }}$ and $\gamma_{\text {sup }}$ their respective coefficients. In other words, we have:

$$
R\left(a ; \mathbf{y}, \mu, \mu^{\prime}\right)=\alpha a^{2}+\beta a+\gamma
$$




$$
\begin{aligned}
& R_{\text {inf }}\left(a ; \widetilde{\mathbf{y}}, \boldsymbol{\varepsilon}, \mu, \mu^{\prime}\right)=\alpha_{\text {inf }} a^{2}+\beta_{\text {inf }} a+\gamma_{\text {inf }} \\
& R_{\text {sup }}\left(a ; \widetilde{\mathbf{y}}, \boldsymbol{\varepsilon}, \mu, \mu^{\prime}\right)=\alpha_{\text {sup }} a^{2}+\beta_{\text {sup }} a+\gamma_{\text {sup }}
\end{aligned}
$$

These coefficients depend on $\mu$ and $\mu^{\prime}$, as well on $\mathbf{y}$ (for $\alpha, \beta, \gamma$ ) and $\widetilde{\mathbf{y}}$ and $\varepsilon$ (for the other coefficients). We do not explicitly write this dependence until the last part of our discussion.

Using (8) we see that the quadratic function of $a$ :

$$
\left(\alpha_{i n f}-\alpha\right) a^{2}+\left(\beta_{i n f}-\beta\right) a+\gamma_{i n f}-\gamma
$$

is negative or zero; hence it takes a non-positive value for $a=0$, and has a non-positive discriminant:

$$
\begin{array}{r}
\gamma_{i n f}-\gamma \leq 0 \\
\left(\beta_{i n f}-\beta\right)^{2} \leq 4\left(\alpha_{i n f}-\alpha\right)\left(\gamma_{i n f}-\gamma\right)
\end{array}
$$

As $\left(\beta_{\text {inf }}-\beta\right)^{2} \geq 0$, Equations (11) and (12) above imply that $\alpha_{\text {inf }}-\alpha \leq 0$, and that:

$$
\beta_{i n f}-\delta_{i n f} \leq \beta \leq \beta_{i n f}+\delta_{i n f}
$$

for $\delta_{i n f}=2 \sqrt{\left(\alpha_{i n f}-\alpha\right)\left(\gamma_{i n f}-\gamma\right)}$.

We now suppose that $\alpha_{i n f}>0$. As $\alpha_{\text {inf }}$ is computable from $\widetilde{y}_{k}, \widetilde{y}_{k}^{\prime}, \varepsilon_{k}$ and $\varepsilon_{k}^{\prime}$, one can practically check if this condition is met. If it is not the case, our bound can not be used. We expect that if the metamodel error is not too large, we will have $\alpha_{\text {inf }} \approx \alpha$ and, as $\alpha>0$, the hypothesis $\alpha_{\text {inf }}>0$ is realistic.

So, under this supplementary assumption, we have:

$$
\underset{a}{\operatorname{argmin}} R\left(a ; \mathbf{y}, \mu, \mu^{\prime}\right)=-\frac{\beta}{2 \alpha} \geq-\frac{\beta_{i n f}+\delta_{i n f}}{2 \alpha_{i n f}}
$$

Now using the second part of (8) and the same reasoning on the non-positive quadratic function of $a: R\left(a ; \mathbf{y}, \mu, \mu^{\prime}\right)-R_{\text {sup }}\left(a ; \widetilde{\mathbf{y}}, \varepsilon, \mu, \mu^{\prime}\right)$, we find that: $\alpha \leq \alpha_{\text {sup }}$, and: $\beta_{\text {sup }}-\delta_{\text {sup }} \leq \beta \leq \beta_{\text {sup }}+\delta_{\text {sup }}$. Hence,

$$
\underset{a}{\operatorname{argmin}} R\left(a ; \mathbf{y}, \mu, \mu^{\prime}\right) \leq-\frac{\beta_{\text {sup }}-\delta_{\text {sup }}}{2 \alpha_{\text {sup }}}
$$

where $\delta_{\text {sup }}=2 \sqrt{\left(\alpha-\alpha_{\text {sup }}\right)\left(\gamma-\gamma_{\text {sup }}\right)}$. This comes without supplementary assumptions, because $\alpha_{\text {sup }} \geq \alpha$ and $\alpha>0$, as the minimum of $R\left(\cdot ; \mathbf{y}, \mu, \mu^{\prime}\right)$ exists (even if the case $\alpha=0$ can seldom occur due to sampling fluctuations, the hypothesis $Y$ not constant a.s. ensures that increasing $N$ and/or changing the sample will lead to a case where $\alpha>0$ ).

As we clearly have $\delta_{\text {inf }}$ and $\delta_{\text {sup }}$ smaller than (or equal to) $\widehat{\delta}:=2 \sqrt{\left(\alpha_{\text {inf }}-\alpha_{\text {sup }}\right)\left(\gamma_{\text {inf }}-\gamma_{\text {sup }}\right)}$, we deduce that:

$$
-\frac{\beta_{\text {inf }}\left(\mu, \mu^{\prime}\right)+\widehat{\delta}\left(\mu, \mu^{\prime}\right)}{2 \alpha_{\text {inf }}\left(\mu, \mu^{\prime}\right)} \leq \underset{a}{\operatorname{argmin}} R\left(a ; \mathbf{y}, \mu, \mu^{\prime}\right) \leq-\frac{\beta_{\text {sup }}\left(\mu, \mu^{\prime}\right)-\widehat{\delta}\left(\mu, \mu^{\prime}\right)}{2 \alpha_{\text {sup }}\left(\mu, \mu^{\prime}\right)}
$$


where we have made explicit the dependencies in $\mu$ and $\mu^{\prime}$.

To finish, it is easy to see that we have:

$$
\overline{\mathcal{P}}:=[\overline{\widetilde{y}}-\bar{\varepsilon} ; \overline{\widetilde{y}}+\bar{\varepsilon}] \ni \bar{y}
$$

and:

$$
\overline{\mathcal{P}}^{\prime}:=\left[\overline{\widetilde{y}^{\prime}}-\bar{\varepsilon}^{\prime} ; \overline{\bar{y}^{\prime}}+\bar{\varepsilon}^{\prime}\right] \ni \bar{y}^{\prime}
$$

(where $\overline{\widetilde{y}}, \overline{\widetilde{y}^{\prime}}, \bar{\varepsilon}$ and $\overline{\varepsilon^{\prime}}$ denote, respectively, the empirical means of $\left(\widetilde{y}_{k}\right)_{k},\left(\widetilde{y}_{k}^{\prime}\right)_{k},\left(\varepsilon_{k}\right)_{k}$ and $\left.\left(\varepsilon_{k}^{\prime}\right)_{k}\right)$ so that:

$$
\min _{\mu \in \overline{\mathcal{P}}, \mu^{\prime} \in \overline{\mathcal{P}}^{\prime}}\left(-\frac{\beta_{\text {inf }}\left(\mu, \mu^{\prime}\right)+\widehat{\delta}\left(\mu, \mu^{\prime}\right)}{2 \alpha_{\text {inf }}\left(\mu, \mu^{\prime}\right)}\right) \leq \widehat{S}_{i}=\underset{a}{\operatorname{argmin}} R\left(a ; \mathbf{y}, \mu, \mu^{\prime}\right) \leq \max _{\mu \in \overline{\mathcal{P}}, \mu^{\prime} \in \overline{\mathcal{P}}^{\prime}}\left(-\frac{\beta_{\text {sup }}\left(\mu, \mu^{\prime}\right)-\widehat{\delta}\left(\mu, \mu^{\prime}\right)}{2 \alpha_{\text {sup }}\left(\mu, \mu^{\prime}\right)}\right.
$$

Hence, (5) is verified with:

$\widehat{S}_{i}^{m}=\min _{\mu \in \overline{\mathcal{P}}, \mu^{\prime} \in \overline{\mathcal{P}}^{\prime}}\left(-\frac{\beta_{\text {inf }}\left(\mu, \mu^{\prime}\right)+\widehat{\delta}\left(\mu, \mu^{\prime}\right)}{2 \alpha_{\text {inf }}\left(\mu, \mu^{\prime}\right)}\right), \quad \widehat{S}_{i}^{M}=\max _{\mu \in \overline{\mathcal{P}}, \mu^{\prime} \in \overline{\mathcal{P}}^{\prime}}\left(-\frac{\beta_{\text {sup }}\left(\mu, \mu^{\prime}\right)-\widehat{\delta}\left(\mu, \mu^{\prime}\right)}{2 \alpha_{\text {sup }}\left(\mu, \mu^{\prime}\right)}\right)$

It is clear that $\widehat{S}_{i}^{m}$ and $\widehat{S}_{i}^{M}$ are computable without knowing the $y_{k}$ s and $y_{k}^{\prime} \mathrm{s}$.

In practice, we compute approximate values of $\widehat{S}_{i}^{m}$ and $\widehat{S}_{i}^{M}$ by replacing the min and max over $\overline{\mathcal{P}} \times \overline{\mathcal{P}^{\prime}}$ by the min and max over a finite sample $\Xi \subset \overline{\mathcal{P}} \times \overline{\mathcal{P}^{\prime}}$.

\subsection{Metamodel error in index estimation: a smoothed alter- native}

In this section, we present a more computationally-intensive, but more precise, alternative to the bound described in the previous section. This alternative is interesting when $\varepsilon$ is moderately small with respect to $f$, as, in this case, the former bound was not sharp enough to be useful in practice.

We begin by noting that since:

$$
\forall k=1, \ldots, N \quad y_{k} \in\left[\widetilde{y}_{k}-\varepsilon_{k} ; \widetilde{y}_{k}+\varepsilon_{k}\right] \text { and } y_{k}^{\prime} \in\left[\widetilde{y}_{k}^{\prime}-\varepsilon_{k}^{\prime} ; \widetilde{y}_{k}^{\prime}+\varepsilon_{k}^{\prime}\right],
$$

we certainly have:

$$
\min _{\mathbf{z} \in \mathcal{Z}} \psi_{i}(\mathbf{z}) \leq \widehat{S}_{i} \leq \max _{\mathbf{z} \in \mathcal{Z}} \psi_{i}(\mathbf{z})
$$

where:

$\mathbf{z}=\left(z_{1}, \ldots, z_{N}, z_{1}^{\prime}, \ldots, z_{N}^{\prime}\right), \quad \mathcal{Z}=\prod_{k=1}^{N}\left[\widetilde{y}_{k}-\varepsilon_{k} ; \widetilde{y}_{k}+\varepsilon_{k}\right] \times \prod_{k=1}^{N}\left[\widetilde{y}_{k}^{\prime}-\varepsilon_{k}^{\prime} ; \widetilde{y}_{k}^{\prime}+\varepsilon_{k}^{\prime}\right]$

and $\psi_{i}(\mathbf{z})$ is the Monte-Carlo sensitivity index estimator for variable $i$ using $\mathbf{z}$ as function evaluations sample:

$$
\psi_{i}(\mathbf{z})=\frac{\overline{z z^{\prime}}-\bar{z} \overline{z^{\prime}}}{\overline{z^{2}}-\bar{z}^{2}},
$$


which satisfies $\psi_{i}\left(y_{1}, \ldots, y_{N}, y_{1}^{\prime}, \ldots, y_{N}^{\prime}\right)=\widehat{S}_{i}$, where $\left\{y_{k}\right\}$ and $\left\{y_{k}^{\prime}\right\}$ are defined in (2) and (3).

Minimizers and maximizers of optimization problems in (16) often display a very irregular (nonsmooth) behavior (as a function of the sampled inputs $\left\{\mathbf{x}_{1}, \ldots, \mathbf{x}_{N}, \mathbf{x}_{1}^{\prime}, \ldots, \mathbf{x}_{N}^{\prime}\right\}$ ), even for a smooth output $f$. This lead to overly pessimistic bounds in (16). To overcome this difficulty, we propose to ensure smoothness of the solution by introducing a penalty factor $\lambda \geq 0$ and to take:

$$
S_{i}^{m, \lambda}=\psi_{i}\left(\mathbf{z}^{m, \lambda}\right) \quad S_{i}^{M, \lambda}=\psi_{i}\left(\mathbf{z}^{M, \lambda}\right)
$$

where:

$$
\mathbf{z}^{m, \lambda}=\underset{\mathbf{z} \in \mathcal{Z}}{\operatorname{argmin}}\left(\psi_{i}(\mathbf{z})+\lambda \Pi(\mathbf{z})\right) \quad \mathbf{z}^{M, \lambda}=\underset{\mathbf{z} \in \mathcal{Z}}{\operatorname{argmax}}\left(\psi_{i}(\mathbf{z})-\lambda \Pi(\mathbf{z})\right)
$$

for $\Pi(\mathbf{z})$ a (non-negative) indicator of the smoothness of the function which take $\mathbf{z}$ as values when evaluated on the input sample $\left\{\mathbf{x}_{1}, \ldots, \mathbf{x}_{N}, \mathbf{x}_{1}^{\prime}, \ldots, \mathbf{x}_{N}^{\prime}\right\}$. For instance, one may take:

$$
\Pi(\mathbf{z})=\frac{1}{2 N} \sum_{k=1}^{2 N}\left(z_{k}-z_{k}^{S}\right)^{2}
$$

with $\left\{z_{k}^{S}\right\}$ a kernel-smoothed version of $\left\{z_{k}\right\}$ :

$$
z_{k}^{S}=\frac{\sum_{k^{\prime}=1}^{2 N} K\left(\frac{\left\|\mathbf{x}_{k^{\prime}}-\mathbf{x}_{k}\right\|}{h}\right) z_{k^{\prime}}}{\sum_{k^{\prime}=1}^{2 N} K\left(\frac{\left\|\mathbf{x}_{k^{\prime}}-\mathbf{x}_{k}\right\|}{h}\right)}
$$

for $K$ an appropriate kernel (preferably with compact support, for reasons of efficiency of the implementation), $h$ a suitable bandwidth (in the sum we have set $\mathbf{x}_{k+N}=\mathbf{x}_{k}^{\prime}$ for $\left.k=1, \ldots, N\right)$, and $\|\cdot\|$ the Euclidean norm on $\mathbf{R}^{p}$. The main difficulty is that these optimization problems are in potentially large dimension $2 N$. However, the gradient of the objective functions are analytically available - under (18) and (19) - and can be cheaply evaluated, so we choose to use a L-BFGS quasi-Newton algorithm, as implemented in L-BFGS-B ([25]), to solve problems (17).

Concerning the choice of $\lambda$ and $h$, we opt for the following: $h$ should be chosen so as to have a reasonable proportion (say, 1 to 5 percent) of "neighbor" points (that is to say, couples of points in $\left\{\mathbf{x}_{k}\right\}_{k=1, \ldots, N}$ having a significantly nonzero value on the kernel $K$ ). For the choice of $\lambda$ : we plot, for a chosen $h$ held fixed, the length $\left|S_{i}^{M, \lambda}-S_{i}^{m, \lambda}\right|$ as function of $\lambda$, and then choose $\lambda$ to be the abscissa of the bottom-left corner of the "L-shaped" curve obtained.

\subsection{Combined confidence intervals}

In Sections 2.2 and 2.3, we have seen how to separately assess sampling error and metamodel error. To take both sources of error into account simultaneously, we propose using bootstrap confidence intervals (see Section 2.1) by 
calculating $B$ bootstrap replications of $\widehat{S}_{i}^{m}$ and $\widehat{S}_{i}^{M}$, where, for $b=1, \ldots, B$ each bootstrap pair $\left(\widehat{S}_{i}^{m}[b] ; \widehat{S}_{i}^{M}[b]\right)$ is computed using $\left(\widetilde{y}_{k}\right)_{k \in L_{b}},\left(\widetilde{y}_{k}^{\prime}\right)_{k \in L_{b}}$ as surrogate output samples, and associated error bounds $\left(\widetilde{\varepsilon}_{k}\right)_{k \in L_{b}},\left(\widetilde{\varepsilon}_{k}\right)_{k \in L_{b}}$, where $L_{b}$ is a list of $N$ integers sampled with replacement from $\{1, \ldots, N\}$. Here the bounds $\widehat{S}_{i}^{m}$ and $\widehat{S}_{i}^{M}$ can be computed using either the method described in Section 2.2, or the one described in Section 2.3.

The BC bootstrap confidence interval procedure (see Section 2.1) can then be used to produce $(1-\alpha)$-level confidence intervals $\left[\widehat{S}_{i, \alpha / 2}^{m} ; \widehat{S}_{i, 1-\alpha / 2}^{m}\right]$ for $S_{i}^{m}$, and $\left[\widehat{S}_{i, \alpha / 2}^{M} ; \widehat{S}_{i, 1-\alpha / 2}^{M}\right]$ for $S_{i}^{M}$. We then take as combined confidence interval of level $1-\alpha$ for $S_{i}$ the range $\left[\widehat{S}_{i, \alpha / 2}^{m} ; \widehat{S}_{i, 1-\alpha / 2}^{M}\right]$. This interval accounts for sampling and metamodels error simultaneously: let, for $b=1, \ldots, B, \widehat{S}_{i}[b]$ be the $b^{t h}$ bootstrap replication, computed using the true outputs $\left(y_{k}\right)_{k \in L_{b}}$. As, for each $b$, we have:

$$
\widehat{S}_{i}^{m}[b] \leq \widehat{S}_{i}[b] \leq \widehat{S}_{i}^{M}[b],
$$

it follows that

$$
\widehat{S}_{i, \alpha / 2}^{m} \leq \widehat{S}_{i, \alpha / 2}, \text { and } \widehat{S}_{i, 1-\alpha / 2} \leq \widehat{S}_{i, 1-\alpha / 2}^{M},
$$

where $\widehat{S}_{i, \alpha / 2}$ and $\widehat{S}_{i, 1-\alpha / 2}$ are the endpoints of the $(1-\alpha)$ BC-confidence interval computed using $\left(\widehat{S}_{i}[b]\right)_{b}$ as replications. It follows that $\left[\widehat{S}_{i, \alpha / 2} ; \widehat{S}_{i, 1-\alpha / 2}\right]$ is contained in $\left[\widehat{S}_{i, \alpha / 2}^{m} ; \widehat{S}_{i, 1-\alpha / 2}^{M}\right]$ and, hence, the level of the latter interval is greater than the level of the former. As the level of the $\left[\widehat{S}_{i, \alpha / 2} ; \widehat{S}_{i, 1-\alpha / 2}\right]$ is (asymptotically) equal to $1-\alpha$, the level of the combined confidence interval $\left[\widehat{S}_{i, \alpha / 2}^{m} ; \widehat{S}_{i, 1-\alpha / 2}^{M}\right]$ should theoretically be, by design, greater than $1-\alpha$.

\section{Applications}

\subsection{Application to a reduced basis metamodel}

The reduced basis method $([15,16])$ can be applied when the output is a functional of the discretized solution of an elliptic or hyperbolic partial differential equation. This method comes with efficient and rigorous error bounds. Due to the intrusive nature of the reduced basis approach and "problem-dependent" considerations made during the construction of the metamodel, one can expect fastly-convergent, tight and fully justified error bounds for the sensitivity indices.

In this section, we test our combined confidence interval procedure described in Sections 2.2 and 2.4, and compare it with Monte-Carlo estimation on the full model (with bias-corrected bootstrap to assess sampling error). Our criteria for comparison are the CPU times needed to compute the intervals and the lengths of these intervals (the smaller the better).

In all our tests we take $\alpha=.05$ and $B=2000$ bootstrap replications. 


\subsubsection{Model set-up}

Let $u$, a function of space $x \in[0 ; 1]$ (note that space variable $x$ is unrelated to input parameter vector $\mathbf{x})$ and time $t \in[0, T](T>0$ is a fixed (i.e., known) parameter) satisfying the viscous Burgers' equation:

$$
\frac{\partial u}{\partial t}+\frac{1}{2} \frac{\partial}{\partial x}\left(u^{2}\right)-\nu \frac{\partial^{2} u}{\partial x^{2}}=\psi
$$

where $\nu \in \mathbf{R}_{*}^{+}$is the viscosity, and $\psi \in C^{0}\left([0, T], L^{2}(] 0,1[)\right)$ is the source term.

For $u$ to be well-defined, we also prescribe initial value $u_{0} \in H^{1}(] 0,1\left[\right.$ ) (ie. $u_{0}$ is in the Sobolev space of square-integrable functions whose first derivative is square-integrable), and continuous boundary values $b_{0}, b_{1} \in C^{0}([0, T])$.

The initial $u_{0}$ and boundary values $b_{0}$ and $b_{1}$ are parametrized the following way:

$$
\begin{array}{clrl}
b_{0}(t) & =\left(u_{0 m}\right)^{2}+\sum_{l=1}^{n\left(b_{0}\right)} A_{l}^{b_{0}} \sin \left(\omega_{l}^{b_{0}} t\right) & b_{1}(t) & =u_{0}(1)+\sum_{l=1}^{n\left(b_{1}\right)} A_{l}^{b_{1}} \sin \left(\omega_{l}^{b_{1}} t\right) \\
\psi(t, x) & =\psi_{m}+\sum_{l=1}^{n_{T}(\psi)} \sum_{p=1}^{n_{S}(\psi)} A_{l p}^{\psi} \sin \left(\omega_{l}^{\psi T} t\right) \sin \left(\omega_{p}^{\psi S} x\right) & u_{0}(x) & =\left(u_{0 m}\right)^{2}+\sum_{l=1}^{n\left(u_{0}\right)} A_{l}^{u_{0}} \sin \left(\omega_{l}^{u_{0}} x\right)
\end{array}
$$

The values of the angular frequencies $\omega_{l}^{b_{0}}, \omega_{l}^{b_{1}}, \omega_{l}^{\psi T}, \omega_{p}^{\psi S}$ and $\omega_{l}^{u_{0}}$, as well as their cardinalities $n\left(b_{0}\right), n\left(b_{1}\right), n_{T}(\psi), n_{S}(\psi)$ and $n\left(u_{0}\right)$ are fixed (known), while our uncertain parameters, namely: viscosity $\nu$, coefficients $\psi_{m}$ and $u_{0 m}$, and amplitudes $\left(A_{l}^{b_{0}}\right)_{l=1, \ldots, n\left(b_{0}\right)},\left(A_{l}^{b_{1}}\right)_{l=1, \ldots, n\left(b_{1}\right)},\left(A_{l p}^{\psi}\right)_{l=1, \ldots, n_{T}(\psi) ; p=1, \ldots, n_{S}(\psi)}$ and $\left(A_{l}^{u_{0}}\right)_{l=1, \ldots, n\left(u_{0}\right)}$ live in some Cartesian product of intervals $\mathcal{P}$ defined by:

$$
\begin{gathered}
\mathcal{P}=\left\{\mathbf{x}=\left(\nu, A_{1}^{b_{0}}, \ldots, A_{n\left(b_{0}\right)}^{b_{0}}, A_{1}^{b_{1}}, \ldots, A_{n\left(b_{1}\right)}^{b_{0}}, \psi_{m}, A_{11}^{\psi}, A_{12}^{\psi}, \ldots, A_{1, n_{S}(\psi)}^{\psi},\right.\right. \\
\left.\left.A_{2,1}^{\psi}, \ldots, A_{2, n_{S}(\psi)}^{\psi}, \ldots, A_{n_{T}(\psi), n_{S}(\psi)}^{\psi}, u_{0 m}, A_{1}^{u_{0}}, \ldots, A_{n\left(u_{0}\right)}^{u_{0}}\right)\right\}
\end{gathered}
$$

The solution $u=u(\mathbf{x})$ depends on the parameter vector $\mathbf{x}$ above.

The "full" model is obtained by discretizing the initial-boundary value problem, using a discrete time grid $\left\{t_{k}=k \Delta t\right\}_{k=0, \ldots, T / \Delta t}$, where $\Delta t>0$ is the time step, and, space-wise, using $\mathbf{P}^{1}$ Lagrange finite elements built upon an uniform subdivision of $[0 ; 1]:\left\{x_{i}=i / \mathcal{N}\right\}$, for $i=0, \ldots, \mathcal{N}$. Our full output is:

$$
f(\mathbf{x})=f(u(\mathbf{x}))=\frac{1}{\mathcal{N}} \sum_{i=0}^{\mathcal{N}} u\left(t=T, x=x_{i}\right)
$$

The reduced basis method is then applied to yield a surrogate solution $\widetilde{u}$ of (20), as well as an error bound $\varepsilon_{u}$ on $u$. The reader can refer to [13] for full 
details on discretization, reduction and derivation of the $\mathrm{RB}$ error bound for this model. The main idea of the reduced basis method is to project $u$ onto a well-chosen subspace of $X$ whose dimension $n$ (the reduced basis size) is smaller than the dimension of the finite element space that can be used to solve (20) numerically.

In our numerical experiments, we take $\mathcal{N}=60, \Delta t=.01, T=.05, n_{S}(\psi)=$ $n_{T}(\psi)=n\left(b_{0}\right)=n\left(b_{1}\right)=0, n\left(u_{0}\right)=1, \omega_{1}^{u_{0}}=0.5, A_{1}^{u_{0}}=5$ and $\psi_{m}=1$.

The two input parameters are independent and uniformly sampled. The table below contains the ranges for them, and also the "true" values of the sensitivity indices, which have been calculated (in more than $14 \mathrm{~h}$ CPU time) using a Monte-Carlo simulation with large sample size $N=4 \times 10^{6}$ (so as to $\mathrm{BC}$ bootstrap confidence intervals of length $<10^{-2}$ ) on the full model:

\begin{tabular}{|c|c|c|}
\hline Parameter & Range & Confidence interval for sensitivity index \\
\hline$\nu$ & {$[1 ; 20]$} & {$[0.0815 ; 0.0832]$} \\
$u_{0 m}$ & {$[0 ; 0.3]$} & {$[0.9175 ; 0.9182]$} \\
\hline
\end{tabular}

We then take:

$$
S_{\nu}=0.082 \text { and } S_{u_{0 m}}=0.918
$$

as "true" sensitivity values.

\subsubsection{Empirical coverage of combined confidence intervals}

We test the performance of our combined confidence intervals by assessing their empirical coverage. This empirical coverage is measured by computing, for each input variable, 100 combined confidence intervals (each time with different input sample), and counting the proportion of intervals containing the true values in (22). The reduced basis is built using POD-based procedure [13] and reduced basis size is $n=9$; Monte-Carlo sample size is $N=300$. Results are gathered in the table below:

\begin{tabular}{|c|c|c|}
\hline Parameter & Mean confidence interval & Empirical coverage \\
\hline$\nu$ & {$[0.0139 ; 0.2083]$} & 0.91 \\
$u_{0 m}$ & {$[0.8421 ; 0.9491]$} & 0.87 \\
\hline
\end{tabular}

\subsubsection{Convergence benchmark}

Figure 1 shows the lower $\widehat{S^{m}}$ and upper $\widehat{S^{M}}$ bounds (defined in Section 2.2) for different reduced basis sizes (hence different metamodel precisions) but fixed sample of size $N=300$, as well as the bootstrap confidence intervals computed using the procedure presented in Section 2.4. This figure exhibits the fast convergence of our bounds to the true value of the sensitivity index as the reduced basis size increases. We also see that the part of the error due to sampling remains constant, as sample size stays the same. 

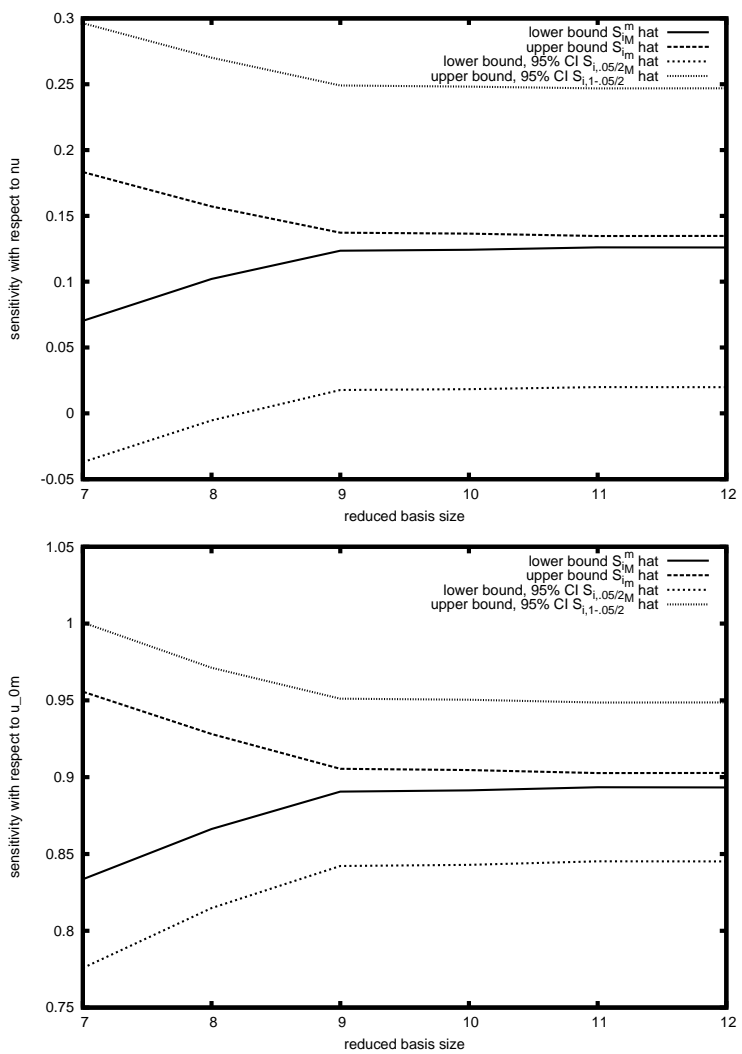

Figure 1: Convergence benchmark for sensitivity indices estimation in the Burgers' model, left: variable $\nu$, right: variable $u_{0 m}$. We plotted, for a fixed sample size $N=300$, estimator bounds $\widehat{S}^{m}$ and $\widehat{S}^{M}$ defined in (2.2), and endpoints $\widehat{S}_{i, .025}^{m}$ and $\widehat{S}_{i, 1-.025}^{M}$ of the $95 \%$ confidence interval, for different reduced basis sizes. 


\subsubsection{Choice of $n$ and $N$}

In practice, one wants to estimate sensitivity indices with a given precision (ie. to produce $(1-\alpha)$-level confidence intervals with prescribed length), and has no a priori indication on how to choose $N$ and $n$ to do so. Moreover, for one given precision, there may be multiple choices of suitable couples $(N, n)$, balancing between sampling and metamodel error. Increasing $N$ and/or $n$ will increase the overall time for computation and improve the precision of the calculation (thanks to reduction in sampling error for increased $N$, or reduction in metamodel error for increased $n$ ).

We wish to choose the best compromise, that is, the one that gives the smallest computation time; for the reduced basis method, this time is roughly proportional to $N \times n^{3}$ (as a number proportional to $N$ of linear systems of size $n$ have to be solved). On the other hand, the length of the combined confidence interval can be modelled as the sum of two terms:

$$
\frac{Z_{\alpha}}{\sqrt{N}}+\frac{C}{a^{n}}
$$

where $Z_{\alpha}, C$ are positive constants and $a>1$ is a constant. The first term accounts for sampling error; its form is heuristically deduced from central limit theorem. The second accounts for metamodel error; its exponential decay is backed up by numerical experiments as well as theoretical works (e.g. [3]). The parameters $Z_{\alpha}, C$ and $a$ can be found by doing "calibration runs" with a fixed small sample size $N$ and different reduced basis sizes $n_{1}, \ldots, n_{K}$, and by regressing the obtained confidence interval lengths using (23).

Minimizing the computation time under the constraint that error is equal to a given "target precision" leads to a straightforward constrained optimization problem $(P)$, which can be solved, after estimation of $Z_{\alpha}, C$ and $n$, to find (after rounding to the closest integer) the optimal $n$ and $N$ for a desired precision.

As an example, we choose, using the same Burgers model with the same parameters than before, a target precision of $p=.02$. The solution to the constrained optimization problem $(P)$ is $n \approx 11$ and $N \approx 22000$.

Conducting the combined confidence interval estimation with these parameters give intervals

[0.0659997;0.0937285] for sensitivity index for $\nu$, and $[0.914266 ; 0.926452]$ for sensitivity with respect to $u_{0 m}$. These confidence intervals have mean length:

$$
\frac{1}{2}(0.0937285-0.0659997+0.926452-0.914266)=0.0199575 \approx 0.02
$$

as desired. 


\subsubsection{Full-scale example}

We now present an example with more parameters. We take $\mathcal{N}=300$, $\Delta t=.01, T=.05, n(\psi)=0, n\left(u_{0}\right)=5, n\left(b_{0}\right)=n\left(b_{1}\right)=2, \psi_{m}=1$, $\omega_{1}^{u_{0}}=.5, \omega_{2}^{u_{0}}=1, \omega_{3}^{u_{0}}=1.5, \omega_{4}^{u_{0}}=2, \omega_{5}^{u_{0}}=2.5, \omega_{1}^{b_{0}}=\omega_{1}^{b_{1}}=.3$ and $\omega_{2}^{b_{0}}=\omega_{2}^{b_{1}}=.6$. Input parameters are assumed independent and uniformly distributed in the ranges below:

\begin{tabular}{|c|c|}
\hline Parameter(s) & Range \\
\hline$\nu$ & {$[1 ; 20]$} \\
\hline$u_{0 m}, A_{i}^{u_{0}}(i=1, \ldots, 5)$ & {$[0 ; 0.2]$} \\
\hline$A_{1}^{b_{0}}$ & {$[1 ; 1.3]$} \\
\hline$A_{2}^{\frac{1}{b_{0}}}$ & {$[0 ; 0.2]$} \\
\hline$A_{i}^{b_{1}}(i=1,2)$ & {$[0 ; 0.2]$} \\
\hline$f_{m}$ & {$[0 ; 0.2]$} \\
\hline
\end{tabular}

We used a reduced basis of size $n=13$, built using a POD snapshot ensemble of size $\# \Xi=200$. The combined confidence intervals obtained, for $N=$ 7000 , have mean length $\approx 0.08$. Their computation required $N \times(p+$ $1)=91000$ calls to the metamodel; these calls took $85 \mathrm{~s}$ of CPU time. By extrapolating the time necessary to generate the 200 (full) model evaluations for the POD snapshot, we found that creating bootstrap confidence intervals of length 0.08 using the full model would require approximately $514 \mathrm{~s}$. The use of a metamodel hence enabled a $6 x$ speedup.

\subsection{Application to a RKHS interpolation metamodel}

\subsubsection{RKHS interpolation and error bound}

We now briefly recall the RKHS (reproducing kernel Hilbert space) interpolation method, and refer to [20] for the details. RKHS interpolation is known to be (see [21]) equivalent to interpolation by Kriging, also known as Gaussian process metamodelling. Using $n$ evaluations of $f$, one can build a training sample consisting of $n$ input-output pairs $\mathcal{D}=\left\{\left(\mathbf{x}_{1}, y_{1}\right), \ldots,\left(\mathbf{x}_{n}, y_{n}\right)\right\}$, where $\left\{\mathbf{x}_{1}, \ldots, \mathbf{x}_{n}\right\}$ is the experimental design and $y_{i}=f\left(\mathbf{x}_{i}\right)$ for $i=1, \ldots, n$. Let $R(\cdot, \cdot)$ be a positive definite kernel; the RKHS interpolator of $\mathcal{D}$ with respect to $R$ is:

$$
\widetilde{f}(\mathbf{x})=\mathbf{k}(\mathbf{x})^{T} \Sigma_{S}^{-1} \mathbf{y}_{S}
$$

where:

$$
\begin{gathered}
\Sigma_{S}=\left(\begin{array}{cccc}
R\left(\mathbf{x}_{1}, \mathbf{x}_{1}\right) & R\left(\mathbf{x}_{1}, \mathbf{x}_{2}\right) & \ldots & R\left(\mathbf{x}_{1}, \mathbf{x}_{n}\right) \\
R\left(\mathbf{x}_{2}, \mathbf{x}_{1}\right) & R\left(\mathbf{x}_{2}, \mathbf{x}_{2}\right) & \ldots & R\left(\mathbf{x}_{2}, \mathbf{x}_{n}\right) \\
\vdots & \vdots & \ddots & \vdots \\
R\left(\mathbf{x}_{n}, \mathbf{x}_{1}\right) & R\left(\mathbf{x}_{n}, \mathbf{x}_{2}\right) & \ldots & R\left(\mathbf{x}_{n}, \mathbf{x}_{n}\right)
\end{array}\right) \\
\mathbf{k}(\mathbf{x})=\left(R\left(\mathbf{x}_{1}, \mathbf{x}\right), \ldots, R\left(\mathbf{x}_{n}, \mathbf{x}\right)\right)^{T}, \quad \mathbf{y}_{S}=\left(y_{1}, \ldots, y_{n}\right)^{T}
\end{gathered}
$$


In practice, $R$ is assumed to belong to a parametrized family; for instance, $R$ is a gaussian kernel:

$$
R(\mathbf{x}, \mathbf{y})=\exp \left(-\sum_{j=1}^{p} \theta_{j}\left(x_{j}-y_{j}\right)^{2}\right)
$$

whose parameters $\theta_{1}, \ldots, \theta_{p}$ are estimated from $\mathcal{D}$ by minimizing some contrast function.

If $f$ belongs to the RKHS associated with the $R$ kernel, there exist a constant $C$, depending only on $f$ and $R$, so that:

$$
|f(\mathbf{x})-\tilde{f}(\mathbf{x})| \leq C \sqrt{\sigma_{Z}^{2}(\mathbf{x})}
$$

where:

$$
\sigma_{Z}^{2}(\mathbf{x})=R(\mathbf{x}, \mathbf{x})-\mathbf{k}(\mathbf{x})^{T} \Sigma_{S}^{-1} \mathbf{k}(\mathbf{x})
$$

We propose to estimate the constant $C$, which is, up to a multiplicative constant, the norm of $f$ in the RKHS associated with $R$, by:

$$
\widehat{C}=\max _{i=1, \ldots, n^{\tau}} \frac{\left|f\left(\mathbf{x}_{i}^{\tau}\right)-\widetilde{f}\left(\mathbf{x}_{i}^{\tau}\right)\right|}{\sqrt{\sigma_{Z}^{2}\left(\mathbf{x}_{i}^{\tau}\right)}}
$$

where $\left\{\mathbf{x}_{i}^{\tau}\right\}_{i=1, \ldots, n^{\tau}}$ is a "test sample" that does not contain any point of the experimental design (so as to ensure that $\sigma_{Z}^{2}\left(\mathbf{x}_{i}^{\tau}\right) \neq 0$ for all $i=1, \ldots, n^{\tau}$ ). For a continuous $f$, one easily sees that $\widehat{C}$ converges, as $\left\{\mathbf{x}_{i}^{\tau}\right\}$ fills the parameter set $\mathcal{P}$, to the smallest $C$ so that (25) holds.

\subsubsection{Application to Ishigami function}

We test our method using a RKHS interpolation metamodel based on the Ishigami function:

$$
f\left(X_{1}, X_{2}, X_{3}\right)=\sin X_{1}+7 \sin ^{2} X_{2}+0.1 X_{3}^{4} \sin X_{1}
$$

for $\left(X_{j}\right)_{j=1,2,3}$ i.i.d. uniform in $[-\pi ; \pi]$.

The analytical values of sensitivities are known:

$$
S_{1}=0.3139, \quad S_{2}=0.4424, \quad S_{3}=0 .
$$

The experimental design of $\mathcal{D}$ have been generated using maximin latin hypercube samples (using maximinLHS of $\mathrm{R}$ lhs package ([4]). The $\mathrm{R}$ package mlegp ([5]) is used as our RKHS interpolation/Kriging toolbox.

Smoothing parameters are set to: $\lambda=.6, h=.2$. We computed, for different learning sample sizes $n$, the empirical coverages and mean lengths of the combined confidence intervals (with the "smoothed" alternative described in Section 2.3). Monte-Carlo sample size is $N=1000$, and $B=300$ bootstrap 
replicates are computed. Finally, as Sobol indices are always bounded by 0 and 1 , the reported confidence interval is the intersection of the computed interval with $[0 ; 1]$. Results are given in the table below:

\begin{tabular}{|c|c|c|c|c|}
\hline Learning sample size $n$ & Variable & Mean 95\% combined conf. int. & Mean length & Coverage \\
\hline 110 & $X_{1}$ & {$[0.207 ; 0.759]$} & 0.552 & 0.94 \\
110 & $X_{2}$ & {$[0.0169 ; 0.561]$} & 0.545 & 0.98 \\
110 & $X_{3}$ & {$[0 ; 0.356]$} & 0.357 & 1 \\
130 & $X_{1}$ & {$[0.262 ; 0.626]$} & 0.364 & 0.83 \\
130 & $X_{2}$ & {$[0.083 ; 0.491]$} & 0.408 & 0.93 \\
130 & $X_{3}$ & {$[0 ; 0.256]$} & 0.257 & 1 \\
160 & $X_{1}$ & {$[0.274 ; 0.509]$} & 0.235 & 0.92 \\
160 & $X_{2}$ & {$[0.216 ; 0.486]$} & 0.27 & 0.92 \\
160 & $X_{3}$ & {$[0 ; 0.180]$} & 0.18 & 1 \\
\hline
\end{tabular}

The bound described in Section 2.2, while faster to compute, did not give interesting results (ie. the produced intervals were larger than $[0 ; 1]$ ), even for large $n$.

We compared these results with the ones obtained with CompModSA, a software package implementing the methodology described in [23]. We used as parameters: surface $=$ 'mlegp' (Kriging metamodel), n.mc . T=0 (we do not want any total index computation), n.mc. $\mathrm{S}=1000$ (sample size), n . samples=1 (one run), and $\mathrm{n} . \mathrm{CI}=300$ (generate confidence intervals using 300 bootstrap replications). We contributed a patch for CompModSA, available at

http://ljk.imag.fr/membres/Alexandre. Janon/compmodsa.php, which adds to sensitivity the option CI.S, set to TRUE to compute bootstrap confidence intervals for the main effect index. The results, for a learning sample of size $n=160$, are shown hereafter:

\begin{tabular}{|c|c|c|c|}
\hline Variable & Mean 95\% Combined conf. int. & Mean length & Coverage \\
\hline$X_{1}$ & {$[0.0164222 ; 0.218084]$} & 0.202 & 0.02 \\
$X_{2}$ & {$[0.0877679 ; 0.351651]$} & 0.264 & 0.11 \\
$X_{3}$ & {$[0.00750793 ; 0.173349]$} & 0.166 & 0.82 \\
\hline
\end{tabular}

This comparison clearly shows that our method is able to account for metamodel error, so as to keep the actual coverage of the produced confidence interval close to the expected one. We have been unable to fully compare our approach with the one of [14], as the lengths of the confidence interval obtained with this approach were not available; however we can state that, in this example, our method produces intervals of correct coverages for every variable and every training set size we have tested.

\section{Conclusion and perspectives}

We presented a methodology to quantify the impact of the sampling error and the metamodel error on the sensitivity indices computation, when 
the metamodel provides a pointwise error bound (or, at least, an error indicator) on the output of interest. Sampling error is handled by a classic bootstrap procedure, while metamodel error is managed using bounds on the sensitivity index estimator. Quantification of those two types of errors permits a certification on the performed estimation We applied our method on two types of metamodels: intrusive (reduced basis) and non-intrusive (RKHS interpolation). On the applications we see that our approach performs well both for reduced-basis and RKHS interpolation. Our method can also be applied with other metamodels, as soon as an assessment for pointwise metamodel error exists.

Acknowledgements We wish to thank Jean-Claude Fort for a suggestion which we exploited to perform our computation of the least square metamodel-induced error bound. We also thank Anestis Antoniadis and Ingrid Van Keilegom for advice on bootstrap methodology, and Robert Miller for language remarks. We finally thank anonymous referees for helpful comments about the overall presentation of the paper. - This work has been partially supported by the French National Research Agency (ANR) through COSINUS program (project COSTA-BRAVA nANR-09-COSI-015).

\section{References}

[1] G. Archer, A. Saltelli, and I. Sobol. Sensitivity measures, ANOVA-like techniques and the use of bootstrap. Journal of Statistical Computation and Simulation, 58(2):99-120, 1997.

[2] S. Boyaval, C. Le Bris, Y. Maday, N. Nguyen, and A. Patera. A reduced basis approach for variational problems with stochastic parameters: Application to heat conduction with variable robin coefficient. Computer Methods in Applied Mechanics and Engineering, 198(41-44):31873206, 2009.

[3] A. Buffa, Y. Maday, A. Patera, C. Prud'homme, and G. Turinici. A priori convergence of the greedy algorithm for the parametrized reduced basis. Mathematical Modelling and Numerical Analysis, 2009.

[4] R. Carnell. lhs: Latin Hypercube Samples, 2009. R package version 0.5.

[5] G. M. Dancik. mlegp: Maximum Likelihood Estimates of Gaussian Processes, 2011. R package version 3.1.2.

[6] B. Efron. Nonparametric standard errors and confidence intervals. Canadian Journal of Statistics, 9(2):139-158, 1981. 
[7] B. Efron and R. Tibshirani. Bootstrap methods for standard errors, confidence intervals, and other measures of statistical accuracy. Statistical science, 1(1):54-75, 1986.

[8] B. Efron and R. Tibshirani. An introduction to the bootstrap. Chapman \& Hall/CRC, 1993.

[9] M. Grepl, Y. Maday, N. Nguyen, and A. Patera. Efficient reducedbasis treatment of nonaffine and nonlinear partial differential equations. Mathematical Modelling and Numerical Analysis, 41(3):575-605, 2007.

[10] M. Grepl and A. Patera. A posteriori error bounds for reduced-basis approximations of parametrized parabolic partial differential equations. Mathematical Modelling and Numerical Analysis, 39(1):157-181, 2005.

[11] J. Helton, J. Johnson, C. Sallaberry, and C. Storlie. Survey of samplingbased methods for uncertainty and sensitivity analysis. Reliability Engineering \& System Safety, 91(10-11):1175-1209, 2006.

[12] T. Homma and A. Saltelli. Importance measures in global sensitivity analysis of nonlinear models. Reliability Engineering 83 System Safety, 52(1):1-17, 1996.

[13] A. Janon, M. Nodet, and C. Prieur. Certified reduced-basis solutions of viscous Burgers equations parametrized by initial and boundary values. Preprint available at http://hal.inria.fr/inria-00524727/en, 2010, submitted.

[14] A. Marrel, B. Iooss, B. Laurent, and O. Roustant. Calculations of sobol indices for the gaussian process metamodel. Reliability Engineering 8 System Safety, 94(3):742-751, 2009.

[15] N. Nguyen, K. Veroy, and A. Patera. Certified real-time solution of parametrized partial differential equations. Handbook of Materials Modeling, pages 1523-1558, 2005.

[16] A. Quarteroni, G. Rozza, and A. Manzoni. Certified reduced basis approximation for parametrized partial differential equations and applications. Math. Industry, 2011.

[17] A. Saltelli. Making best use of model evaluations to compute sensitivity indices. Computer Physics Communications, 145(2):280-297, 2002.

[18] A. Saltelli, K. Chan, and E. Scott. Sensitivity analysis, 2000.

[19] T. J. Santner, B. Williams, and W. Notz. The Design and Analysis of Computer Experiments. Springer-Verlag, 2003. 
[20] R. Schaback. Mathematical results concerning kernel techniques. In Prep. 13th IFAC Symposium on System Identification, Rotterdam, pages 1814-1819. Citeseer, 2003.

[21] M. Scheuerer, R. Schaback, and M. Schlather. Interpolation of spatial data - a stochastic or a deterministic problem ? 2011.

[22] I. Sobol. Global sensitivity indices for nonlinear mathematical models and their Monte Carlo estimates. Mathematics and Computers in Simulation, 55(1-3):271-280, 2001.

[23] C. Storlie, L. Swiler, J. Helton, and C. Sallaberry. Implementation and evaluation of nonparametric regression procedures for sensitivity analysis of computationally demanding models. Reliability Engineering Es System Safety, 94(11):1735-1763, 2009.

[24] K. Veroy and A. Patera. Certified real-time solution of the parametrized steady incompressible Navier-Stokes equations: Rigorous reduced-basis a posteriori error bounds. International Journal for Numerical Methods in Fluids, 47(8-9):773-788, 2005.

[25] C. Zhu, R. Bryd, and J. Nocedal. L-BFGS-B: Algorithm 778: L-BFGS$B$, FORTRAN routines for large scale bound constrained optimization, 1997. 